\title{
LA COMUNIDAD CULTURAL IBEROAMERICANA Y EL NACIONALISMO ESPANTOL
}

\author{
Isidro Sepúlveda Murioz \\ Universidad Nacional de Educación a Distancia. Madrid. España.
}

Las múltiples formulaciones nacionalistas hispanas se han definido en gran medida en un fenómeno de retoalimentación, señalando la existencia de cada una de las conceptualizaciones nacionales en relación contraria a las otras. Surgido al cobijo de la existencia del Estado, el nacionalismo español del siglo XIX basó su identificación de carácter liberal en los principios de la soberanía nacional triunfantes a partir de la Revolución Francesa; por contra, los llamados nacionalismo periféricos (denominación de origen geográfico que fue acogida con grandes dosis de simbolismo y posteriormente de disciplencia) se desarrollaron en las últimas décadas del siglo pasado con la utilización de los principios del Volkgeist de origen germánico. Este nacionalismo de carácter cultural -aun con la misma vocación política que el anterioraportó a la ideología nacionalista importantes elementos identificadores: el particularismo o hecho diferencial, el idioma como arca telúrica del espíritu, la historia como prueba y testimonio de la existencia secular de la nación, la cultura -impregnada de las más altas cimas del pensamiento y la creación nacional a la vez que reunión de las costumbre y el folklore propios-, la religión - motivo de identificación contraria respecto al exterior- $y$, en su extremo más forzado, la raza o la elevación del particularismo de la nación a la categoría biológica. El mantenimiento de discursos a dos niveles radicalmente distintos facilitó la expansión de los emergentes nacionalismos periféricos, por lo que el nacionalismo español, sin abandonar la formulación anterior, acabó radicando en su propia identidad cultural la legitimidad de su existencia. 
La evolución aquí esbozada de estas exposiciones nacionalistas y el debate sobre la identidad nacional, lo que la historiografía conoce como problema de España, se produjo en el periodo de la Restauración, encontrando en el año 1898 la culminación de sus planteamientos y a la vez el punto inicial de nuevas orientaciones. A pesar que el estudio del nacionalismo en España se ha venido conjugando generalmente en plural, tan intenso como la formación de un espíritu nacional catalán, vasco o gallego (por no hablar del valenciano, andaluz o aragonés) se llevó acabo desde el último tercio del siglo XIX un fuerte debate formativo en el interior del nacionalismo español.

El trabajo presente, continuación de otros anteriores, ${ }^{1}$ analiza en tan amplio campo de estudio la trascendencia de uno de los componentes básicos del nacionalismo español: la continuidad cultural española en América y su trascendencia en la conformación de una identidad trasatlántica como imaginario de afirmación nacionalista española, lo que se ha conceptualizado como pan-hispanismo. ${ }^{2}$

El pan-hispanismo aquí analizado no debe ser interpretado como movimiento político tendente a la conformación de una plataforma estatal hispano-americana, basada en un conjunto de instituciones y sujeta a una letra constitucional; ${ }^{3}$ independientemente de ser presentado así por algunos de sus apologetas más conspicuos, las formulaciones pan-hispanistas son esencial e intrínsecamente españolas y deben ser interpretadas como un proceso de recreación de componentes identificadores del nacionalismo español, cuya principal característica es su instrumentalización como argamasa de la identidad colectiva y a la vez como proyección exterior de la misma.

\section{IDENTIDAD Y NACIONALISMO}

La utilización de una identificación nacionalista se encuentra inescusablemente basada en la definición de una identidad colectiva, que a su vez es conformada por unos principios generadores. El estudio de estos principios es pues uno de los principales campos de trabajo para abordar el complejo fenómeno nacionalista; José Acosta ha señalado la necesidad de cuatro elementos centrales conformadores de una nación: el dominio de una sociedad sobre sus condiciones de existencia; la voluntad política unitaria; la oposición de esa sociedad respecto a un exterior distinto y amenazante; y la presencia de una identidad colectiva. ${ }^{4}$

Sobre cuáles son los componentes que crean y sostienen esa identidad colectiva existe un amplio debate, en primer lugar por la interpretación de su aparición como origen de la existencia de esa sociedad. Paradigmas de estas 
interpretaciones pueden ser la utilización del origen remoto e independiente de la lengua vascuence para asegurar la existencia de la nación vasca; o la elevación de la autodenominación a la categoría de constitución, como el rastreo del nombre de España en el reino visigodo, hecho ya denunciado por Américo Castro y Maraval.5 Debate que también se produce por la indefinición en el uso de algunos conceptos genéricos como raza o cultura, cortados trasversalmente por interpretaciones etnicistas, culturalistas, filológicas, antropológicas e historicistas, por lo que parece más necesario una definición de los componentes que su mera enumeración nominativa.

Independientemente del momento en que se ha señalado su primera utilización como elementos conformadores de la identidad de una sociedad, Kedourie centraba su atención sobre la raza, la cultura y la historia (para él incorporación del nacionalismo alemán en la primera mitad del siglo XIX) ${ }^{6}$; a las que sin duda alguna se deben añadir la religión y una categoría muy abstracta - si no lo fueran en absoluto las anteriores - que podría denominarse providencia o misión, tan trascendentes en el desarrollo de nacionalismos como el estadounidense, en su Manifest Destiny, o el judío, con el secular empeño de vuelta a la Tierra Prometida. Aunque en diferente medida, este último componente está presente en todos los nacionalismos, ya sea para cumplir con la aspiración de constituir una entidad política (alcance ésta la categoría de estado o no), sea para trazar un proyecto de futuro, sea para ampliar la base social y consolidar el entramado de la comunidad ante cualquier tipo de amenaza externa.

Estos componentes refuerzan la aparición y la expansión del fenómeno nacionalista y, a su vez, éste incrementa el valor de aquéllos, en un proceso de fortalecimiento mutuo que en los casos más radicales alcanza a confirmar estados totalitarios y establecer sistemas fundamentalistas. Los ejemplo de esto son demasiados numerosos, tanto en el pasado como en el día a día presente. Penúltimos ejemplos de este proceso de fortalecimiento entre el nacionalismo y sus componentes primigeneos es el resurgimiento espectacular de la Iglesia ortodoxa en la Rusia de Yelsin, la "limpieza étnica" en la guerra múltiple en Bosnia (donde operan, con una efectividad dramática, los componentes históricos, religiosos, culturales y, solo en último término, étnicos) o la batalla del idioma en Puerto Rico, en su intento de diferenciacióndistanciamiento respecto a Estados Unidos. 


\section{UNA IDENTIDAD TRASNACIONAL ESPANYOLA}

Como no podía ser de otro modo el nacionalismo español participa de estos componentes identificadores. No es el propósito de este trabajo tratar de analizar cada uno de los elementos que dan significación y singularidad al nacionalismo español. El objetivo principal es mostrar cómo estos elementos fueron interpretados hasta hallar paralelismo y continuidad con los de otras naciones americanas y con ello señalar la existencia de una comunidad hispano-americana.

El nacionalismo español ha variado sustancialmente, al compás de los acontecimientos nacionales e internacionales, su interpretación de la comunidad hispano-americana. Aunque los intentos de establecer las bases de esta comunidad se remontan a épocas anteriores a los mismos procesos americanos de independencia, no fue hasta el segundo tercio del siglo XIX, teniendo presente los procesos de unificación alemana e italiana, cuando se evidenciaron esfuerzos tendente a reforzar los anteriores lazos hispanoamericanos. ${ }^{\text {? }}$

A partir de la década de los ochenta se generó el movimiento que de modo definitivo acabó subrayando el componente vocacional español hacia América como parte inseparable de la identidad nacional española. ${ }^{8} \mathrm{El}$ hispano-americanismo, compuesto por diversas corrientes con distinto proyecto de futuro y hasta cierto punto opuestas, significó en su variante exterior el intento de España de formar un imperio que, por la impotencia de su capacidad material, solo podía aspirar a tener una dimensión espiritual (tesis panhispanista); o establecer un bloque de países con vocación unionista (tesis progresista); o conformar una suerte de Estados Unidos hispano-americanos (tesis latinoamericanista). En su variante interna, el hispano-americanismo estuvo escindido en sus objetivos; mientras una corriente pretendía poner de manifiesto las bases que condujeron a España a su etapa de máximo esplendor para ponerlas nuevamente en práctica (tesis conservadora), otra rompía con los sueños imperiales y, señalando las carencias, veía en la proyección hacia América el medio de regenerar España.?

Lo que explicita la voluntad de utilización de la dimensión hispana en América por el nacionalismo español es tanto el desarrollo simultáneo del hispano-americanismo con el resto de nacionalismos ibéricos, como el paralelismo en la conformación de sus componentes. Dos afios antes de la creación de la Lliga de Catalunya (1887) fue creada en Madrid la Unión IberoAmericana, la más importante asociación americanista hasta la guerra civil. 1892, el mismo año en el que se aprobaban en Manresa las Bases per la Constitucid Regional Calana y Sabino Arana publicaba Bizcaya por su independencia. Cuatro glorias patrias, se celebraba con más pompa que efectividad 
el IV Centenario del Descubrimiento de América, apareciendo una amplia publicística pan-hispanista. ${ }^{10} \mathrm{Si}$ pueden señalarse como hitos conformadores de opinión e inicio de la trayectoria nacionalista los escritos de Prat de la Riva y Pere Mutanyola Compendi de doctrina catalanista (1895) y El Partido Carlista y los Fueros Vasko-Navarros (1897) de Sabino Arana, de igual modo deben entenderse las obras de Rafael $\mathrm{M}^{\mathrm{a}}$ de Labra, Rafael Altamita y un gran número de artículos en las revistas Unión Ibero-Americana, Revista Contemporánea y La Ilustración Española y Americana; además de buena parte de la literatura regeneracionista, comenzando por $E l$ problema nacional (1890) de Lucas Mallada; todo ello encontraba eco y reafirmación en América en obras como Nuestra raza (1900) del argentino Ernesto Quesada; La raza cósmica, J. Vasconcelos (20'5).

La radicalización de los supuestos más conservadores del hispano-americanismo durante la II República propició la aparición de la hispanidad; filosofía de estado que supone el punto culminante de identificación entre la dimensión americana de España y las bases del nacionalismo reaccionario español, en sus dimensiones de catolicidad, antiliberalismo, anticomunismo y providencialismo. " Esta radicalización de las hispanidad en buena parte estaba motivada por la extensión de la base social y las exigencias de autogobierno de los nacionalismos, especialmente del catalán y del vasco, entendidos como una negación de España (no habia, no se quería que hubiera, una diferenciación entre el "gran rechazo" aranista y los intentos catalanes o gallegos de reestructuración del estado); esto motivó un movimiento contrario que tomó la forma de una reivindicación de la interprestación providencialista de la historia de España. ${ }^{12}$ Tomada en parte como base por el nuevo régimen franquista e institucionalizado su pensamiento en el Consejo de la Hispanidad y el Instituto de Cultura Hispánica, la hispanidad acabó siendo el portaestandarte de la visión providencialista de la historia de España, elemento legitimador del régimen, plataforma de proyección exterior (especialmente válida en los tiempos de máximo aislamiento diplomático) y valor añadido en las negociaciones con las potencias internacionales (evidente en las mantenidas con Estados Unidos en los años cincuenta y la Comunidad Económica Europea en los sesenta). ${ }^{13}$

Desde el periodo de la transición y con especial ejecución por la administración socialista se ha desarrollado un nuevo proyecto de proyección española hacia América que ha tomado el sonoro nombre de Comunidad Iberoamericana de Naciones (CIAN), heredero de los presupuestos mantenidos por el hispano-americanismo progresista del primer tercio de siglo, aunque su identificación con el nacionalismo español ha dado paso a un internacionalismo de estado cuyos elementos, aun siendo los mismos, son 
interpretados como propios de la comunidad, desplazando su origen por temor a ostentaciones de legitimidad histórica ajenas a los postulantes del proyecto. Arenal y Nájera definen éste en razón de sus principios operativos. El principio de interdependencia marca la necesidad de operar de modo no coyuntural, mediante una política integradora, global y solidaria. El principio de credibilidad, en la base de una política de Estado, por el que se apunta a una ejecución concreta, trascendente, en busca de hechos identificables y posibles. Lo que está directamente conectado con el principio de continuidad, por el que se requiere el diseño de una política global y de aplicación constante, no sujeta a los posibles vaivenes de cambios políticos, económicos o de mera simpatía entre dirigentes. Es por ello también necesario el principio de indiscriminación, basado en una práctica desideologizada, que mantenga en primer lugar la máxima de no intervención en asuntos internos; lo que no puede ser confundido con la indiferencia a la política interna de los distintos Estados. Finalmente, estos autores señalan la necesidad de mantener el principio de "unidad en la diversidad", que propugna la integración respetando las diversidades de cada uno de los miembros y pretende contrastar con anteriores intentos unionistas de tono paternalista y uniformado. ${ }^{14} \mathrm{La}$ Comunidad Iberoamericana de Naciones ha institucionalizado su existencia, siquiera de un modo tan desburocratizado como la articulación de la Conferencia Iberoamericana mediante la celebración de Cumbres de Jefes de Estado y de Gobierno (1991, Guadalajara -México-; 1992, Madrid; 1993, Bahía -Brasil-; 1994, Cartagena -Colombia-; 1995, Argentina). ${ }^{15}$

Tanto el hispano-americanismo como la Comunidad Iberoamericana de Naciones están conformados por proyectos de futuro diferentes, incluso opuestos entre sí. Ya se ha señalado la oposición ideológica y operativa en el interior del movimiento hispano-americanista. En su dimensión institucional la CIAN, además de las tensiones lógicas entre una veintena de estados -algunos con conflictos abiertos con sus vecinos- se encuentra al arbitrio de cambios sustanciales en la dirección de las políticas exteriores de cada uno de los gobiernos; políticamente, como proyecto de acción exterior española, su articulación se encuentra solapada por la preeminencia de la política hacia Europa y muy cuestionada por los partidos de la oposición parlamentaria. Sólo la permanencia ininterrumpida en el gobierno de un mismo partido y un mismo líder ha permitido el mantenimiento de una línea constante, si bien con una ondulación que tiene su punto de inflexión a la baja en el año 1992.

A pesar de ello, la presencia de un pan-hispanismo como componente básico del nacionalismo español se ha mantenido a lo largo de más de un 
siglo y bajo todos los gobiernos y regímenes existentes. Las evidentes diferencias ideológicas se han manifestado más sobre la instrumentalización de este componente que sobre su propio carácter, que ha permanecido en cuanto tal inalterable en su formulación y en los elementos que lo llenan de contenido. $Y$ es precisamente en esos elementos donde se percibe con claridad la vocación transatlántica del pan-hispanismo y su sólo aparentente contradicción de vocación transnacional. Un examen más detenido de esos elementos pondrá de manifiesto tales afirmaciones.

\section{ELEMENTOS OPERATIVOS DEL PAN-HISPANISMO}

Dentro de los intentos de alcanzar una definición y un corpus operativo en el estudio del nacionalismo se sostiene la pretensión explícita de constituir una nación como estado o alcanzar un estado cuerpo de nación. $\mathrm{El}$ pan-hispanismo presente en el nacionalismo español mediante distintos movimientos (hispano-americanismo), filosofias de estado (hispanidad) o proyectos de política exterior (CIAN), se encuentra en el segundo caso: el esfuerzo un estado en alcanzar una nueva dimensión nacional, dentro de la redefinición ocasionada por la crisis finisecular.

Esta identidad de nacionalismo de estado conlleva varias consecuencias. La primera de las cuales es la posible duda conceptual sobre su carácter, dada la relativa facilidad con la que el pan-hispanismo podría ser interpretado como imperialismo. ${ }^{16}$ Aunque en buena parte de su discurso establece formulaciones con recurrentes paralelismos en otras proclamas para la expansión de los estados, la interpretación que hacen los propios inspiradores de su discurso evidencia el carácter nacional de la dimensión hispana en América, en ocasiones como un mero discurso interno. Esta dimensión refuerza la proyección exterior española, pero sobre todo fortalece la identidad interna, con carácter excluyente respecto a los nacionalismo periféricos. Si Unamuno, Ortega, Marañón o Ruiz Jiménez subrayaron la pertenencia a una misma comunidad de bonaerenses, limeños, mexicanos y valencianos, su objetivo implícito era ignorar las diferencias excluyentes entre catalanes, vascos, gallegos y castellanos.

La segunda consecuencia de este nacionalismo de estado es el reducido trasfondo social, el mínimo apoyo que se encuentra en exíguas bases sociales que pudieran apoyar su materialización. Que el pan-hispanismo se basara más en dimensiones culturales y espirituales que en materiales (desde el mero territorio al establecimiento de mercados) produjo una abstracción que imposibilitó su seguimiento por amplios sectores sociales, quedando reducido su formulación y ejecución a reducidos círculos intelectuales y políticos. 
La tercera consecuencia, directo origen de la anterior, se encuentra en la necesidad de un sentimiento comunitario como base para la formulación de la comunidad hispano-americana, alcanzara ésta dimensión política o no. Dicho sentimiento se percibe en la participación en unos elementos conformadores de identidad nacional, que posteriormente serán analizados. Entre estos, el pan-hispanismo subrayó la importancia de la raza y sobre todo de la lengua. Elementos tanto más importantes para basar la existencia en la gemeinschaft ibero-americana como para ignorar las peculiaridades nacionales dentro de la gessellschaft española.

En su introducción al estudio de la utilización de la identidad cultural en las relaciones internaciones, Reszler y Browning señalaron diferentes elementos constituyentes de esa identidad, interesantes para la determinación de los nacionalismos en cuanto a su oposición externa: la religión destaca por su factor de integración al tiempo que símbolo de oposición entre culturas; la comunicación en una misma lengua; el recuerdo del pasado en la participación de una historia común; la raza como valor de integrador social; las convergencias entre sistemas políticos en armonía ideológica; y finalmente la cultura, entendida como circulación de ideas y expansión de estímulos, tanto integradores como excluyentes. ${ }^{17}$

Los elementos constitutivos de la identidad hispano-americana concitaron tal interés que su defensa y promoción fueron señalados como la misión que americanos y españoles debían llevar a cabo. ${ }^{18}$ Estos elementos fueron la protección y fomento del idioma castellano, la permanencia de la religión católica, la prevención de trastornos contra el orden social establecido y la contención del expansionismo estadounidense. Aunque no explícita (sólo en algunos autores se llega a proponer directamente) existía una misión subyacente a todas las anteriores y era la formulación de una suerte de unión, de variado tipo pero más allá de la mera conciencia común espiritual. Semejante problema surge a la hora de definir el concepto de raza, pues posteriores interpretaciones étnicas y biológicas, han distorsionado la interpretación globalizadora que en su época se dio al término.

Cómo interpretaron estos distintos proyecto los elementos conformadores de la identidad de comunidad hispano-americana: los anteriormente señalados raza, cultura, historia, religión y providencia o misión. Se centra este análisis en los tres elementos más ampliamente utilizados por el pan-hispanismo y que más evidencian el paralelismo entre éste y los nacionalismo periféricos: la historia, la raza y el idioma. 


\section{a) La continuidad del pasado común}

La base histórica sobre la que operaba el pan-hispanismo para probar la existencia de la comunidad hispano-americana no podía ser otra cosa que los tres siglo del periodo colonial, cuando la España oficial (representada en la monarquia indiana) estaba constituida en su mayor extensión por los territorios americanos. El modo de interpretar esta base histórica dividió las formulaciones del americanismo español. Por un lado conllevó la prolongación de posiciones publicísticas arraigadas en la interpretación providencialistas del pasado, que proyectaba hasta el presente y hacia un futuro inmediato objetivos que se habían llevado a cabo con la colonización española (interpretación pan-hispanista); o se denunciaba el providencialismo - dejando de lado la mayor parte del período colonial, juzgado como la causa que llevó a España a un callejón sin salida-, sostenedor de un anquilosamiento nacional y de una falta de contemporaneidad española (tesis progresista). Personificando ambas corrientes, Menéndez Pelayo (abriendo una línea de pensamiento que llegó hasta Vegas Latapié o Víctor Pradera) encontró en los siglos XVI y XVII la esencia de la labor de España en América y con ello de la España imperial; al contrario, para Labra la culminación de la hermandad hispano-americana era la elaboración conjunta de la Constitución de Cádiz.

Pero esta participación en un pasado español común tenía su contraposición en el juicio sobre ese pasado, radicalmente distinto de un lugar a otro; no sólo entre España y las naciones americanas, sino también dentro de cada unidad, dependiendo en gran medida de las concepciones ideológicas de los autores. Los argumentos más críticos a la labor de España en América acabaron encuadrándose en lo que, con tanto éxito como polémica, Julián Juderías denominó leyenda negra:

"Por leyenda negra entendemos el ambiente creado por los fantásticos relatos que acerca de nuestra Patria han visto la luz pública en casi todos los países; las descripciones grotescas que se han hecho siempre del carácter de los espanoles como individuos y como colectividad; la negación o, por, lo menos, la ignorancia sistemática de cuanto nos es favorable $\mathrm{y}$ honroso de las diversas manifestaciones de la cultura y del arte; las acusaciones que en todo tiempo se han lanzado contra España, fundándose para ello en hechos exagerados, mal interpretados falsos en su totalidad. [...] En una palabra, entendemos por leyenda negra, la leyenda de la España inquisitorial, ignorante, fanática, incapaz de figurar entre los pueblos cultos, lo mismo ahora que antes, dispuesta siempre a las represiones violentas; enemiga del progreso $y$ las innovaciones". 19

Para el pan-hispanismo la leyenda negra era un "muro de odio resultante de una historia envenenada de calumnias". ${ }^{20}$ Difícilmente se podía llevar a cabo un acercamiento mientras se mantuviera en pie todo el conglo- 
merado de odios y malquerencias históricas; ya que, en palabra de Antonio Goicoechea, "el enmascaramiento de la Historia, la leyenda negra, ha sido uno de los elementos que más han frenado el acercamiento de España e Hispanoamérica". ${ }^{21}$

La leyenda negra de España en América se combatió de tres modos diferentes. El primero y más sencillo fue ignorarla, señalar que era cosa del pasado o descalificarla como fruto del mero odio a España. La segunda postura fue la que pretendió su refutación mediante la construcción de una contraofensiva publicística y en ocasiones historiográfica que trataba de demostrar lo radicalmente contrario: las bondades de la conquista y el desarrollo alcanzado en el período colonial; ese fue el nacimiento de la conocida como leyenda rosa o blanca. El tercer modo de actuar fue el sometimiento al trabajo científico y a las evidencias documentales, lo que además de tiempo conllevó la asumisión de una parte importante de las acusaciones repetidas durante siglos. Sobre la primera postura nada se dirá, pues su descalificación proviene de sus propios argumentos; la segunda importa en cuanto en España y por grandes períodos fue más conocida la leyenda blanca que la negra, siendo usual pasto de discursos e instrumento propagandístico desde instancias oficiales. La tercera posición es sin embargo mucho más importante, pues además de la autoridad ética que presentó, fue la base del desarrollo de la actual historiografía americanista.

Para el pan-hispanismo la piedra angular era la leyenda blanca. Dicha tesis presuponía la inexistencia de unas mínimas culturas americanas con antelación a 1492, demostraba un desconocimiento soberbio del sistema colonial, ensalzaba la labor de la Iglesia en la evangelización del continente y elevaba a los protagonistas más destacados del proceso colonizador a la categoría de héroes de gesta. Para ilustrar esta posición puede tomarse como ejemplo (uno entre centenares en la misma línea) la postura de Valentín Gutiérrez Solana en plena dictadura primorriverista;

"Esparia ha realizado, providencialmente sin duda, la obra más grande que registra la historia: el descubrimiento, conquista y civilización de América. Solamente la raza hispana, esencialmente creadora, pudo llevar a cabo epopeya tan gloriosa, debido a la fe y amor de nuestros antepasados, por que una $y$ otro han sido el fundamento de nuestras glorias. [...] Nadie puede negar que fue providencial el descubrimiento de América. Basta fijarse en la situación que se encontraban aquellos pueblos entonces, sumidos en el fanatismo más grosero, en la ignorancia, en la molicie y en la tiranía más espantosa." 22

En consecuencia, todas las reivindicaciones de la obra de España en América emitidas posteriormente desde las proclamas de la hispanidad eran meras variaciones sobre el mismo tema. 
El pan-hispanismo contemplaba el pasado español en América como una época de depósito de identidad de lo verdadero español. Desde ese posicionamiento América tenía una identidad definida en cuanto España había sido su conformadora; a la vez, España era en sí misma por haber cumplido su misión, más creativa que transformadora. La esencia de lo creado, tanto en América como en la Península, no era otra cosa que una comunidad o, utilizando el concepto de la época, un raza.

\section{b) Comunidad cultural o raza}

Los siglos de historia común y la labor de España en América, desde los presupuestos pan-hispanistas, habían tenido como fruto la conformación de una comunidad o raza hispanoamericana, compuesta por las sociedades de todos los estados iberoamericanos. La constitución de esta raza-comunidad era en sí misma la más rotunda negación de los nacionalismo periféricos; ignorando su participación en ella y negando su existencia dentro de la macro-comunidad transestatal. Una de las más puntuales definiciones del concepto de raza-comunidad manejada por el pan-hispanismo se debe al mexicano Teófilo García:

"Al hablar de Raza manifestamos, ya que no aludimos precisamente al origen común étnico, sino a cierta identidad psicológica que consciente o inconscientemente empuja a una o a varias colectividades a realizar fines semejantes. El punto de enlace, acaso se determine mejor en la esfera de los sentimientos y de las ideas que en la esfera de la estructura orgánica, y aun de los antecedentes históricos. De todas maneras, el estudio y el reconocimiento de este fenómeno nos lleva a la convicción de que, fruto o no de la voluntad, existe afinidad moral positiva, indestructible entre los pueblos ibero-americanos." 23

La defensa de la existencia de una raza hispana transatlántica estaba basada en la creencia previa de un carácter nacional, tomado como entidad estática, apenas mutable en sus manifestaciones más superficiales. Para que España pudiera haber trasplantado su identidad a América era necesario que la tuviera con anterioridad. Por otro lado, que ese carácter nacional injertado en América durante los siglos XVI y XVII fuera el existente a finales del siglo XIX y principios del XX, denotaba otra creencia en la prolongación intemporal de esa identidad, creencia por tanto contraria a cualquier principio dinámico.

Rafael Altamira mantenía que las naciones adquirían a través de los siglos su propio carácter nacional, dando por supuesto que una vez alcanzado éste permanecía inalterado. Para comprender su posición al respecto es 
necesario señalar la publicación en 1902 (escrito según propia declaración entre 1898 y 1900) de Psicología del pueblo español donde aparecía un regeneracionismo reformista que trataba de "formular las líneas generales de nuestro carácter y determinar, quizás, lo que es en él verdaderamente fundamental, a diferencia de lo transitorio y fortuito"; ${ }^{24}$ apoyado en una metodología académico-positivista, su formulación encontraba un principio idealista del "carácter nacional". Esta aparente contradicción entre el método y el resultado fue resuelta con la variación de ese idealismo en la seguridad incuestionable de la existencia de un espiritu nacional; la trasposición del carácter nacional al Estado estaba asumida implícitamente, donde a su vez aparecía asumida la máxima bíblica heredada del maestro Giner: Regnum divisum, desolabitur. ${ }^{25}$ Este planteamiento tuvo acogida en América de la mano de intelectuales positivistas como Manuel A. Bermúdez o Justo Sierra. Como señalara el profesor Ortiz, es necesario vincular la obra de Altamira a la historiografía liberal, instauradora del concepto de nación como sujeto colectivo de proceso histórico; pero la identificación nación =España en un proceso de religación (tomando el concepto de Zubiri) nacionalista, en su obra se amplia a civilización española: lo que de español (que para él es lo esencial) tiene la sociedad americana; ${ }^{26}$ en ello emplea la definición fichtiana de nación como comunidad de cultura. Fue esta asunción lo que dio lugar a la idea de misión española en América. ${ }^{27}$

¿Qué identificaba ese carácter nacional hasta hacer diferente a España de cualquier otra nación? Los rasgos básicos de ese carácter que señalaron los autores españoles fueron concernientes a la psicología, la religión, la etnia o bien fueron definidos en oposición a contrarios. Abundando en las razones psicologicas de la raza hispana, Lucas Mallada - sin así llamarla - se adelantó a Rodó al señalar que se caracterizaba por mantener los valores sobre la racionalidad, el espíritu sobre la materia, la teoría sobre la práctica y la belleza sobre la razón. ${ }^{28}$ En esta exclusión dual, que en principio imposibilitaba a la raza española para su participación plena en el mundo moderno, se vio el principio de su defensa al mantener que las sociedades desarrolladas anglosajonas había puesto los objetivos materiales como grandes fines, lo que inevitablemente les conducía a la desilusión, pues en los propósitos meramente materiales no podía basarse un ideal de civilización válido para el futuro.

Desde planteamientos progresistas, la imbricación del carácter nacional con la prolongación americana y al mismo tiempo la puntual definición de "qué cosa sea lo español", la dio Altamira en una conferencia en 1926:

"Lo espafiol hállase constituido para nosotros de dos partes; una formada por cosas que no se definen, que se sienten; que son inefables, que sólo perciben 
los espíritus preparados originalmente para ello, pero escapan fácilmente a quienes proceden de campos muy lejanos y diferentes del nuestro: cosas, en fin, que tocan a la sentimentalidad de nuestro pais y de nuestra vida...

"[Las segundas] Son las representadas por nuestros ideales colectivos, que hemos incubado y predicado a través de los siglos, y por los grandes hechos que hemos realizado en nuestra historia." 29

Fueron dos los componentes básicos que Altamira expuso para la identificación de esa constitución de lo español-americano: la común historia y el idioma común, para españoles y americanos. ${ }^{30}$ Tal idea estaba presente en gran parte de los autores hispano-americanos, al proceder de la misma esencia de la historiografía nacionalista en su pretensión de equiparar, si no confundir, pueblo, nación y estado. Ya a mediados del siglo XIX Borregó aseguraba:

"La personalidad de los pueblos, a la que los escritores modernos apellidan nacionalidad, la constituye la raza, la lengua y la historia, y dondequiera que estos tres vínculos unan a los hombres, el separarlos es una obra violenta y antiprovidencial." 31

La creencia en la existencia de un carácter nacional español necesitaba la evidencia de la continuidad. Su permanencia inalterable a lo largo de los siglos no probaba su continuidad en América. La piedra de toque al respecto era el período de las emancipaciones. El tema de la independencia planeó siempre a la hora de asegurar la pervivencia de esa prolongación del carácter español en América. Si esta ruptura y separación había conllevado el final de la presencia española en América, o si ésta permanecía en las propias sociedades americanas fue un tema de debate recurrente. Para los autores españoles la respuesta omnipresente, aunque no monocorde, fue la que afirmaba la continuidad de la raza hispana en América. Se señalaba la antigua existencia de una intimidad intelectual y moral entre América y España en el curso de los siglos de vida común, mediante unos lazos que se habían interiorizado hasta conformar el carácter de las repúblicas americanas. ${ }^{32}$ En todo caso, las sociedades que habían conquistado los Estados independientes no eran un reflejo de las pretéritas culturas indias, sino continuidad de la sociedad colonial, herederas del espíritu, las tradiciones y la cultura del período hispánico.33

El período emancipador se contemplaba como fruto de una evolución interna de los pueblos hispánicos, no como una ruptura explícita con las bases que los habian formado. La idea generalmente admitida era que los virreyes y gobernadores españoles habian sido sustituidos por hombres que, aun respondiendo a diferentes patronímicos, eran tan hispanos como ellos, cuando no se contemplaba a los libertadores americanos como herederos o 
continuadores de los conquistadores españoles de tres siglos atrás. ${ }^{34}$ Esta idea queda formal y brillantemente sintetizada en el paralelismo que establece Unamuno entre la personificación del espíritu y del ideal español, don Quijote, y la figura que personifica la voluntad independentista americana, Bolívar.

A esta idea sobre la emancipación como continuidad, le sucede otra de inevitabilidad. Para muchos autores espanoles (y también para muchos americanos) América mantenía la identidad española; en consecuencia sus ciudadanos, lo quisieran o no, pertenecían mediante esa identidad a la raza espańola. Unamuno estudió la influencia que España continuaba ejerciendo en América; la causa de esta influencia en Argentina, superando grandes obstáculos, era la pervivencia de los valores hispanos en las entrañas de la identidad argentina, mientras que otras influencias desaparecían o apenas alcanzaban la superficie. ${ }^{35}$ Argentina fue, debido a la entrada de un fuerte contingente de emigrantes no españoles y a la influencia cultural de Francia entre sus intelectuales, un banco de prueba de la resistencia que el carácter hispano tenía en América; fue Ortega y Gasset, quizá el autor español más influyente en el Plata, el que fijó el juicio más contundente sobre el tema al afirmar que Argentina habĺa sido España e inevitablemente continuaba siéndolo; ${ }^{36}$ la presentación de cualquier razonamiento con posterioridad al enunciado anterior, explicita su propia innecesidad. Valera, uno de los críticos españoles (con Clarín y Unamuno) que más hizo por dar a conocer a autores americanos en la Península, era un claro ejemplo de exasperación ante las confesiones americanas de latinismo. Su idea de lo que él llamó raza española o ibérica fue una de las más evidentes muestras del paternalismo residual que gran parte del hispano-americanismo español mantuvo durante todo el primer tercio de siglo, pasando amplificado por la falta absoluta de perspectiva a la idea de hispanidad.

Un caso muy contrario lo presentó Miguel de Unamuno, para quien "raza tiene un sentido histórico, espiritual y no antropológico, no material". ${ }^{37}$ Todo lo que en Valera era fuerza para la retención de prestigio en Unamuno fue polémica enriquecedora; pocos como él lucharon en España por la igualdad en la estima de americanos y españoles y él fue quien marcó el camino para establecer una de las promociones más sustanciosas del pan-hispanismo: la utilización del idioma como proyección de la personalidad nacional o, como dijera el rector salmantino, de la sangre del espiritu.

Los nacionalismos catalán y vasco vieron pronto la necesidad de manifestar sus ideales en una conmemoración festiva y reivindicativa (Aberri Eguna y Diada); por su parte el hispano-americanismo (y más concretamente su rama del pan-hispanismo nacionalista) también concretó en una fecha 
emblemática la manifestación de sus ideales y sus demandas. Tanto el hecho que se tomó como motivo de conmemoración como el nombre que se le dio al evento son muy significativos del alto valor que tenían para el nacionalismo español: 12 de octubre (descubrimiento de América y celebración de la Virgen del Pilar, Patrona de España), Fiesta de la Raza. De origen privado y concevida en principio como plataforma de manifestación comunitaria hispano-americana y de demandando una mayor dedicación a América por parte de la política exterior española, pronto cayó en la repetición de meros actos folclórico-discursivos, perdiendo todo su carácter reivindicativo. Aunque celebrado el 12 de octubre de forma aislada en 1892, la organización de un festejo anual y el carácter que inicialmente tuvo fue una iniciativa de la Unión Ibero-Americana a partir de 1912. Hasta 1918 el gobierno no decretó oficial la fiesta, cuando ya se festejaba oficialmente en Panamá, Costa Rica, Nicaragua, Brasil, (todos con un carácter distinto, semejante al Columbus Day estadounidense, aunque con posterioridad varió en sentido hispano); la República Dominicana (desde 1912), Guatemala y Puerto Rico (1913, en este caso llamado "Día de las Américas"); Bolivia, Honduras y Paraguay (1914); Ecuador, El Salvador y Uruguay (1915); Argentina y Perú (1917). Colombia decretó fiesta nacional el 12 de octubre el mismo año que España; con posterioridad lo hicieron Venezuela y Chile (1921), Cuba (1922) y México (1928). Durante la II República continuó celebrándose la Fiesta de Raza, ganando en contenidos aunque no en plasmación de objetivos. Los dirigentes franquistas trataron desde un principio de diferenciar su celebración de la del periodo republicano, por lo que transmutó en Fiesta de la Hispanidad, al considerar la anterior "de marcado carácter comunista, democrático y antiespañol". ${ }^{38}$

\section{c) Identidad nacional e idioma.}

La lengua, como almacén de los valores psicológicos de las naciones, fue uno de los elementos más utilizados por el pan-hispanismo; tanto pasa basar la existencia de la comunidad hispano-americana, como para negar la participación de los nacionalismos hispanos, excluyentes desde la perspectiva étnico-lingüística. Por ello la importancia del vínculo idiomático no dejó de ponerse de manifiesto:

"El gran instrumento de unión es el idioma. Es el gran vinculo del hispanismo; el idioma es nuestro gran tesoro anfictiónico, la argamasa del edificio hispánico compuesto de piezas diferentes. [...] El habla española es lo que mantiene en los pueblos hispánicos el sentimiento de universalidad racial, en perjuicio del carácter nacional de cada uno de los componentes. El valor del 
idioma no es sólo, y con esto ya sería mucho, el del medio fácil y grato de comunicación para todas las obras del comercio humano. Es también el gran archivo psicológico que conserva los valores comunes del espíritu" ${ }^{39}$

La utilización de la lengua como elemento de identidad y argamasa nacional se llevó a cabo por el pan-hispanismo de dos modos. El primero fue la constatación de los valores psicológicos que la lengua tenía. Rafael Altamira mantenía que la pureza de Castilla se encontraba en la íntima conexión entre el espíritu y la lengua, por lo que no se podía corromper la lengua con aportaciones foráneas sin que el espíritu perdiera su identidad. Ampliando el ámbito de atención, su juicio alcanzaba validez universal cuando afirmaba que "la lengua es el espíritu de un pueblo". 40 La lengua era el gran archivo psicológico donde los pueblos conservan sus valores comunes; por lo que creaba por sí misma una comunidad intelectual que, en cuanto tal, no podía ser penetrada por quien desconocían la lengua. Pero sin duda fue Unamuno quien sintetizó más afortunadamente esta idea en los conocidos versos de su soneto La lengua: "La Sangre de mi espíritu es mi lengua/ y mi patria es alli donde resuene".

Un segundo modo de utilización se centró en el valor integrador de la lengua. El pan-hispanismo vela en ella el principal medio del que se había servido la España colonizadora para crear de una variedad dispersa de civilizaciones y sociedades una única comunidad integrada. Al pretender reforzar esa comunidad entre España y América se retomaba la utilización del lenguaje; por lo que parecía necesario defenderlo de posible corrupciones. Los principales peligros que se percibían por ello eran las influencias de otras lenguas que por diversos caminos llegaban a América: el francés con el ascendente de su alta cultura sobre la élite americana, el inglés mediante el comercio y el italiano por la masiva inmigración en la zona del Plata.

La caída del castellano, superado en el uso por otra lengua, era visto como una doble amenaza, tanto para la presencia de España en América como para el futuro de la identidad hispano-americana; este último punto muy presente en la publicística americana:

"La raza española corre grave peligro en las naciones hispano-americanas; no solo el cosmopolitismo de estos países, por razón de la influencia inmigratoria, tiende a quitar a aquéllos su carácter típico, sino que del norte para el sur viene poco a poco efectuando una pacffica conquista la enemiga raza sajona de los Estados Unidos".41

De una importancia muy destacada fue la aportación de Unamuno a la conformación de la unidad hispano-americana en torno a la lengua común. Mientras que otros autores pusieron el acento sobre el concepto de raza o identidad cultural entre los pueblos americanos y español, Unamuno 
combatió oportunamente esta opción, declarando en cuantas ocasiones tuvo que la base de la comunidad hispano-americana estaba en la lengua. Una de estas oportunidades, muy significativa, fue la crítica a la obra de Carlos Octavio Bunge Nuestra América, donde el concepto de raza traspasa los límites culturales y de civilización para ahondar en los planteamiento biológicos heredados de Gobineau:

"Me parece que más que con la sangre les va a los sudamericanos el españolismo con la lengua, sangre del espíritu, en la que reciben en potencia todo un modo de pensar y concebir, y con las costumbres y hábitos y tradiciones populares. Quien hable en espafiol pensará en espafiol, quiéralo o no, y aunque ni lo crea ni lo sepa". ${ }^{42}$

Unamuno fue quien más claramente definió el concepto de raza en su sentido espiritual y cultural; raza era el depósito de la tradición histórica y cultural cuya base de expresión y proyección exterior era la lengua. En este sentido la raza "psíquica, la espiritual, la crea la lengua, que es la sangre del espíritu". 43 Para él no existía la pureza de raza, además de que ésta no pueda basarse en criterios fisiológicos, somáticos o materiales; para él la raza es la cultura, y su vehículo: el idioma, la lengua común. La lengua muestra por sí sola, afirmó Unamuno, una visión y un punto de vista del universo, una concepción de la vida y del destino humano y de todas sus manifestaciones, incluidas su concepción filosófica y la religión que profesa:

"La Fiesta de la Raza espiritual espafiola no debe, no puede tener un sentido racista material - de materialismo de raza-, ni tampoco un sentido eclesiástico - de una o de otra iglesia-, y mucho menos un sentido político. Hay que alejar de esa fiesta todo imperialismo que no sea el de la raza espiritual encarnada en el lenguaje. Lenguaje de blancos, y de indios, y de mestizos, y de mulatos; lenguaje de cristianos católicos y no católicos, y de no cristianos, y de ateos; lenguaje de hombres que viven bajo los más diversos regimenes políricos." 44

Mucho más que la sangre, mezclada en América en múltiples cruces, o las costumbres, evolucionadas por la influencia de población india y por la emigración no española, si España había otorgado algo a América había sido una lengua y, mantenida su pervivencia, ese era el principal vínculo de unión entre ambos continentes y por tanto la plataforma más adecuada para fundar la comunidad hispano-americana. La misma idea también fue desarrollada por Rodó, uniendo raza y lengua:

"La persistencia invencible del idioma importa y asegura la del genio de la raza, la del alma de la civilización heredada, porque no son las lenguas ánforas vacías donde puede volcarse indistintamente cualquier sustancia espiritual, sino formas orgánicas inseparables del espiritu que las anima y que manifiesta por ellas".45 


\section{A MODO DE CONCLUSIÓN.}

Según lo hasta aquí expuesto, el pan-hispanismo supuso la pretensión de dotar a la idea de la nación española de una base discursiva de carácter cultural, poniéndola en relación con la proyección exterior histórica del Estado y de la monarquía española. Al reivindicar las dimensiones histórica y cultural, el pan-hispanismo fue por tanto no solo un componente más del nacionalismo español, sino el factor más decisivo para la transformación del carácter del discurso nacionalista, desde unos presupuestos liberales con origen en la Revolución francesa al nacionalismo culturalista de origen germánico, ya utilizado por los nacionalismos y regionalismos hispanos.

El pan-hispanismo surgió como parte del movimiento finisecular de cuestionamiento y redefinición de la identidad espaniola. Con posterioridad acabó conformando un componente esencial del nacionalismo español y, aun reinterpretado durante los diferentes regímenes políticos desde perspectivas en ocasiones radicalmente distintas, ha permanecido incorporado e incuestionado como base misma de la esencia y la identidad nacional española.

Además de encardinador de la formación de una idea trascendental y en ocasiones providencialista de la labor de España en América, el pan-hispanismo ha sido utilizado desde posiciones políticas y culturales muy distintas. Las consecuciones principales han sido su interpretación como valor añadido del peso real de España en el concierto internacional y como instrumento de proyección exterior. Ambas dimensiones han sido utilizadas además por el nacionalismo español tanto de cara al interior -frente a los nacionalismo periféricos-, como en el plano internacional, presentando España como cabeza o portavoz de la comunidad de estados iberoamericanos.

Por último, el pan-hispanismo ha sido motivo de reflexión sobre los elementos constituyentes de esa comunidad iberoamericana, y por extensión - dentro de la dinámica de identificación de las esencias española y americana- de la propia identidad española. Los elementos conformadores de esa identidad tenidos como más importantes coinciden con los inicialmente señalados al apuntar las principales aportaciones del nacionalismo romántico-culturalista de origen germánico: la historia (instrumentalizada por el pan-hispanismo desde el providencialismo al estudio de las instituciones comunes), la raza (tomada como conceptualización de la comunidad cultural, sin connotaciones etnicistas en la gran mayoría de los casos) y la lengua, tenida como el basamento primigenio de la comunidad. 


\section{NOTAS}

1 SEPÚLVEDA, I.: "Nacionalismo espafiol y proyección hacia América: el pan-hispanismo"; BERAMENDI, J. et al. (eds.): Nationalism in Europe: past and present; Santiago de Compostela, Universidade de Santiago, 1994; vol. II, pp. 319-336.

2 SEPÚLVEDA, I.: Comunidad cultural e hispano-americanismo, 1885-1936; Madrid, UNED, 1994; vid. pp. 63-92.

SNYDER, L.L.: Macro-nationalisms. A History of the Pan-Movements, Westport, Conn., Greenwood Press, 1984.

4 ACOSTA SANCHEZ, J.: "Los presupuestos teóricos del nacionalismo y el nuevo ciclo del fenómeno."; Revista de Estudios Pollticos, 77; julio-septiembre de 1992; p. 130.

5 CASTRO, A.: Los españoles: como llegaron a serlo; Madrid, 1965; p.23.- MARAVAL, J.A.: "Notas sobre el origen de Español"; Studia hispanica in honorem R. Lapesa, Madrid, 1974; vol. II, pp. 343354.

6 KEDOURIE, E.: Nacionalismo; Madrid, Centro de Estudios Constitucionales, 1985.

7 VAN AKEN, M.: Pan-hispanism: Its Origin and Developmen to 1866; Berkeley, University of California Press, 1959.- RAMA, J.C.: Relaciones culturales entre España y América Latina en el siglo XIX; México, F.C.E., 1982.- LoPEZ-OCóN, L.: Biografla de 'La América'. Una crónica hispanoamericana del liberalismo democrático español. (1857-1886); Madrid, C.S.I.C., 1987.

8 PIKE, F.B.: Hispanismo, 1898-1936. Spanish Conservatives and Liberals and Their Relations with Spanish America, Notre Dame [Indiana], University of Notre Dame Press, 1971.- MAINER, J.C.: "Un capítulo regeneracionista: el hispanoamericanismo (1892-1923)"; en La doma de la quimera (Ensayos sobre nacionalismo y cultura en Espatia); Bellaterra, Universidad Autónoma de Barcelona, 1988; pp. 87-138.

9 SEPúlVEDA MUÑOZ, I.: Comunidad cultural e hispano-americanismo. Op. cit.

10 Un repertorio amplio puede encontrarse en BERNABEU ALBERT, S.: 1892: El $\mathrm{N}$ Centenario del Descubrimiento de América en España: Coyuntura y Conmemoraciones, Madrid, C.S.I.C., 1987.

11 A diferencia del hispano-americanismo, el número de obras básicas a partir de las cuales se desarrollo la hispanidad es mínimo: Defensa de la Hispanidad de Ramiro de Maeztu y Apologia de la Hispanidad de Mons. Gomá y Tomás; ambas en Ramiro de Maeztu. Obrá ed. de Vicente Marrero; Madrid, Editora Nacional, 1974. GARCLA MORENTE, M.: Orfgenes del nacionalismo españoh Buenos Aires, s.e., 1938. Así como numerosos artículos en la revista Acción Española.

12 GARCIA VILLADA, Z.: El destino de España en la historia universat Madrid, Cultura Espafiola, 1936. La primera edición de la obra apareció por capítulos en Acción Española durante el año 1935.

13 MARTIN ARTAJO, A.: Hacia la Comunidad Hispdnica de Naciones, Madrid, Cultura Hispánica, 1956.- GONZáLEZ CALLEJA, E., LIMóN NEVADO, F.: La Hispanidad como instrumento de combate. Raza e imperio en la Prensa franquista durante la Guerra Civil española Madrid, C.S.I.C., 1988.- DELGADO, L.: Imperio de papel. Acción cultural y politica exterior durante el primer franquismo; Madrid, C.S.I.C., 1992.- ABELLáN, J.L., MONCLúS, A. (Comp.): El pensamiento español contemporáneo y la idea de America, Barcelona, Antropos, 1989; vol. 2.

14 ARENAL, C. del; NAJERA, A.: LaComunidad Iberoamericana de Naciones: pasado, presente $y$ futuro de la olitica iberoamericana de España, Madrid, CEDEAL, 1992; pp. 345-350.

15 SEPúlVEDA MUÑOZ, I.: “Proyectos españoles de proyección hacia América. Pasado y presente"; en Portugal, España y América, Madrid, UNED,1993.

16 SILVA, J.F.: Reparto de América Española y Pan-Hispanismo; Madrid, Librerla Espanola y Extranjera, [1918].- En el famoso Tercer Punto de la declaración doctrinal de la Falange se expli- 
cita: Tenemos vocación de imperio [...] Respecto a los patses de Hispanoamérica, tenderemos a la unificación de su cultura, de intereses económicos y de poder. Espania alega su condición de ge espiritual del mundo bipdnico como titulo de preeminencia en las empresas universales.

RESZLER, A.; BROWNING, A: "Identité culturelle et relations internationales (libres propus sur un gran théme)"; Relations Internationales, 24, 1980; pp. 385-386. Se entiende por identidad cultural "une composante d'un principe d'identité plus vaste téunissant un ensemble de valeurs, de croyances, de traditions et d'aspirations plus ou moins cohérentes et intégrées en fonction d'une finalité éprouvée."

18 PLA, J.: La Mision internacional de la raza hispdnica, Madrid, Javier Morata Ed., 1928.- VASCONCELOS, J.: La Raza Cósmica Misión de la raza iberoamericana; México, Asociación Nacional de Libreros, 1983; (1ªd., 1925).

19 JUDERIAS, J.: La leyenda negra. Estudios acerca de España en el extranjero; Madrid, s.e., 1914.

20 CORRAL, B.: "¿̨or qué España no ha entrado en el corazón de los americanos?"; Unión IberoAmericana, diciembre de 1918; pp. 30-31.

21 GOICOECHEA, A.: La obra pasada y la actual de España en América, III Ciclo de conferencias organizadas por el Centro Gallego de Montevideo; Montevideo, s.e., 1928; p. 13.

22 GUTIERREZ SOLANA, V.: La Gran Familia Hispanoamericana, Madrid, Imp. Hispánica, s.a. [1926]; pp. 6-7.

23 GARCIA, T.: "La raza: Patria, raza y humanidad e iberoamericanismo"; Revista Positiva, 12; México, 1. XII.1901; pp.498-499.

24 ALTAMIRA, R.: Psicologia del pueblo español $3^{a}$ ed, Madrid, Editorial Doncel, 1976.

25 ALTAMIRA, R.: Los elementos de la civilización y del cardcter españoles, $2^{2}$ ed., Losada, Buenos Aires, 1956; pp. 43-55.- La edición desglosa y aumenta algunos capítulos de su Epttome de Historia de España (1927), que refleja la idea mantenida desde su Psicologla del pueblo español (1902).

26 ORTIZ, A.: "Regeneracionismo e historiografla: el mito del carácter nacional en la obra de Rafael Altamira"; en ARBEROLA, A. (Ed.): Estudios sobre Rafael Altamira Alicante, Instituto Juan Gil Albert, 1987; pp. 275-351.

27 Un estudio genérico sobre el uso del concepto raza en la época de Altamira en REBÉRIOUX, M.: "Le mot race au tournant du siècle"; Mots. Les lenguages du politique, 33, déc. 1992; pp. 53-58.Todo el número de esta revista el concepto y la utilización de raza, DONNAFOUS, S.; HERSZBERG, B.; ISRAEL, J.: "Sans distinctio de... Race"; Ibidem.

28 MALLADA, L.: Los males de la patriá, Madrid, M. G. Hernández, 1890; p. 132.

29 Conferencia pronunciada el 20 de diciembre de 1926 en el Centro de Intercambio Intelectual Germano-Español, publicada con el título Cómo concibo yo la finalidad del Hispanoamericanismo, Madrid, Tip. Blass, 1927; pp. 8-10.

30 "De aqul que no pueda haber mejor lección de espafiolismo que la emanada de nuestros grandes escritores, si no la que procede de nuestra historia." ALTAMIRA, R.: La politica de Espafia en América, Valencia, Edeta, 1921; p. 74.

31 BORREGO, A.: De la sinuación y los intereses de España en el movimiento reformador de Europa Madrid, 1841; p. 133.

32 LABRA, R.Mª: El problema hispano-americano; Madrid, 1906; p. 36.

33 MAGARIÑOS, S., PUIGDOLLERS, R: Panhispanismo. Su trascendencia histórica, polttica y social; Prólogo de Rafael Altamira; Barcelona, Ed. Cientifica-Médica, 1926.; p. 32.

34 DOUSSINAGUE, J.M².: "Simón Bolivar, "El Político"; Revista de las Españas, mayo-junio, 1929; pp. 242-244.

35 UNAMUNO, M.: Temas argentinos, Buenos Aires, Instituto de Cultura Espaniola, 1943; p. 173- 
196.

36 ORTEGA Y GASSET, J.: Meditación del pueblo joven; Buenos Aires, Emecé, [1958]; p. 65.- Este volumen contiene ensayos y discursos de Ortega desde 1916, fecha de su primer viaje a Buenos Aires.

37 "Una celebración"; La Nación, Buenos Aires, 3.XII.1922; en Obras Completas, Barcelona, Vergara, 1959-64, 16 vol.; vol. IV, p. 1.072.

38 Paradójicamente la denominación de "Fiesta de la Hispanidad" no se hizo oficial hasta la publicación del Deçcreto de 10 de enero de 1958.- Toda la documentación sobre la organización y nueva orientación del festejo en Archivo de la Agencia Espańola de Coooperación Internacional, Arc. 44, exp. 186.

39 GóMEZ BAQUERO, J.: "Nacionalismo e Hispanismo"; Revista de las Espafias, marzo-abril de 1928; p. 76.

40 ALTAMIRA, R.: España en América; Valencia, Sempere y Cla, 1908; p. 37.

41 QUESADA E.: Nuestra raza; Buenos Aires, Librería Bredahl, 1900; p. 23.

42 UNAMUNO, M.: "De la literatura hispanoamericana" (agosto de 1903); en Obras Completas, vol IV, p. 810.

43 UNAMUNO, M.:"La raza ibero-americana en la gran raza latina"; Unión Ibero-Americana, 1.III.1904; p. 43.

44 UNAMUNO, M. de: "De nuevo la raza" (12.X.1933); en Obras Completas, vol. VI, p. 944.- Para una mayor precisión de su postura ver además los artículos: "La Fiesta de la Raza" y "La comunidad de la Lengua hispánica", en Temas argentinos.

45 RODO, J.E.: "El Genio de la Raza"; en Obras Completas; Madrid, Aguilar, 1967; p. 1.213. 